\title{
DERIVING THE CANONICAL EQUIVALENT CIRCUIT FOR SMALL SIGNAL \& LOW FREQUENCY AC MODEL FOR THE SEPIC AND ZETA PWM DC-DC CONVERTERS WITH TWO-PORT NETWORK (QUADRIPOLES) CIRCUIT ANALYSIS TECHNIQUE
}

\author{
Enio V. Kassick \\ INEP-UFSC: Power Electronics Institute - Federal University of Santa Catarina - P.O. Box 5119, 88049-970 Florianópolis SC \\ Brazil \\ kassick@inep.ufsc.br
}

\begin{abstract}
PWM dc-dc converters operating in Continuous Conduction Mode (CCM) perform similar basic functions despite the topology of which one. The canonical equivalent circuit of small signal \& low frequency ac model permits to analyze converter behavior in a general manner, without the need to make reference to a specific topology. For Buck, Boost, BuckBoost and Cuk topologies the "plug-in" parameters of the canonical model can be easily found just by circuit manipulation using very simple circuit analysis techniques. For the Sepic (Single-Ended Primary Inductor Converter) and Zeta topologies, utilization of the same procedure fails and one cannot arrive to the canonical model. To overcome this barrier, some authors indicate the need to apply circuit synthesis techniques which are not always familiar to many engineers. It will be shown that it is possible to arrive to the canonical model of this two converter topologies by using the wellknown two-port network (quadripoles) circuit analysis technique.
\end{abstract}

Keywords - Canonical Model, PWM CCM DC-DC Converters, Quadripoles, Sepic Converter, Small Signal \& Low Frequency AC Model, Zeta Converter.

\section{INTRODUCTION}

The need for dynamic models of any electronic device is almost mandatory concerning the design of control systems. This is also true for power electronics converters such as PWM CCM dc-dc converters and, as a result, one can find various papers and books dealing with this subject spanning for more than three decades until now-a-days [1]-[18].

Technical literature brings us a lot of different approaches in order to derive such models [1]-[18], but perhaps the most simple and useful is the one based on waveforms averaging.

Dynamic modeling for Buck, Boost, Buck-Boost and Cuk PWM CCM dc-dc converters leading to the canonical equivalent circuit shown in Figure1, starting from the general equivalent electrical circuit derived from the dynamic equations can be easily performed. One just need to refer the voltage \& current sources to the primary side of the cascaded ideal transformers and moving the impedances to the secondary side of those ideal transformers [2] and [7]. After that, one can represent the cascaded ideal transformers by a single equivalent ideal transformer with the according turns ratio.

${ }^{1}$ Manuscript received in 10/10/2011; first review in 16/11/2011. Accepted for publication in 18/11/2011 recommended by the editor in charge Antonio J. Marques Cardoso.
When applying the very same procedure to the Sepic and Zeta PWM CCM dc-dc converters one gets to a dead-end and fails in the attempt to represent these converters by a canonical equivalent circuit.

For these two converters, the general equivalent electrical circuit derived from the dynamic equations present ideal transformers connected in a "circular way", that can be seen as a kind of inner power feedback loop, as shown later in Figure 3 for Sepic converter.

In fact, one can apply circuit synthesis techniques to obtain the fourth-order low-pass filter topology (shown in Figure 1) that matches with the line-to-output and control-tooutput voltage transfer functions of those converters, but it might become a very hard task. Perhaps this is the main reason why the canonical equivalent circuit ac models for those two PWM CCM dc-dc converters are not so often present in technical literature [1]-[19].

This work shows that, in order to overcome this difficulty one can apply the well-known two-port network (quadripole) analysis technique and arrive with ease to the topology and parameters of such fourth-order low-pass filter.

It will be show in details how to do this for PWM CCM dc-dc Sepic converter in order to derive the fourth-order lowpass filter topology and, as a consequence, develop a complete dynamic equivalent circuit. For the PWM CCM dcdc Zeta converter only the final results are presented, because one must just apply the same procedure (mutatis mutandis) to be successful.

\section{CANONICAL EQUIVALENT CIRCUIT DYNAMIC MODEL FOR PWM CCM DC-DC CONVERTERS}

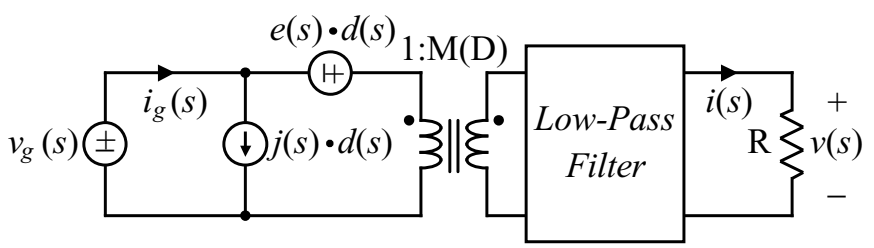

Fig. 1. Dynamical equivalent circuit for PWM CCM dc-dc converters in the canonical form.

The dynamic equivalent small signal \& low frequency ac circuit of PWM CCM dc-dc converters in the canonical form is shown in Figure 1 [2] and [7], where M(D) represents the ideal converter conversion ratio. For Buck, Boost and BuckBoost converters the output low-pass filter is a second order and for Cuk, Sepic and Zeta converters this filter is a fourthorder one.

With this circuit one can represent on the Laplace domain (s-domain) the influence of disturbances in the output voltage 
$v(s)$, related to disturbances on the line (input) voltage $v_{g}(s)$ and on the duty-cycle $d(s)$ [2] as stated by

$$
v(s)=G_{v g}(s) v_{g}(s)+G_{v d}(s) d(s)
$$

It is also possible to represent the influence of disturbances in the input current $i_{g}(s)$, related to variations on the line (input) voltage $v_{g}(s)$ and on the duty-cycle $d(s)$ [2] as stated by

$$
i_{g}(s)=G_{i g}(s) v_{g}(s)+G_{i d}(s) d(s)
$$

Coefficients $e(s)$ and $j(s)$ of the voltage and the current sources (shown in Figure 1) of the canonical circuit model of those converters can easily be obtained [2], as done by

$$
\begin{gathered}
e(s)=\frac{G_{v d}(s)}{G_{v g}(s)} \\
j(s)=G_{i d}(s)-e(s) G_{i g}(s)
\end{gathered}
$$

It's important to stress that the presence of this ac sources accounts for the ac variations in the converters voltages and currents caused by control input variations, represented by $d(s)$, where $G_{v d}(s)$ and $G_{v g}(s)$ are the converter control-tooutput and the converter line-to-output voltage transfer functions, respectively, and $G_{i d}(s)$ and $G_{i g}(s)$ are the duty ratio $d(s)$ to input current $i_{g}(s)$ transfer function and the input admittance or the quotient of the input current $i_{g}(s)$ and the input voltage $v_{g}(s)[2]$.

\section{DYNAMIC EQUATIONS OF THE SEPIC PWM CCM DC-DC CONVERTER} 2.

The PWM CCM dc-dc Sepic converter is shown in Figure

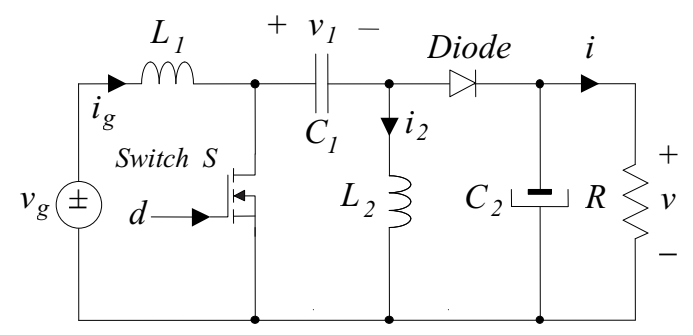

Fig. 2. Sepic PWM CCM dc-dc converter.

The set of dynamic equations, already in s-domain, of this converter can be easily obtained [7] and are presented by

$$
s L_{1} i_{g}(s)=v_{g}(s)-v_{1}(s) D_{c}-v(s) D_{c}+\frac{V_{g}}{D_{c}} d(s)
$$

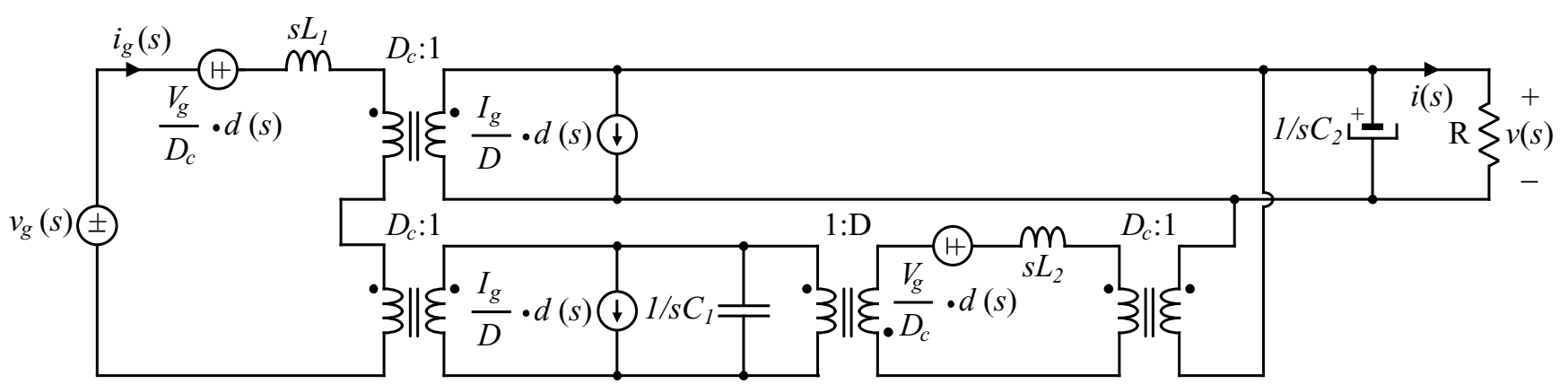

$$
\begin{gathered}
s C_{1} v_{1}(s)=D i_{2}(s)+D_{c} i_{g}(s)-\frac{I_{g}}{D} d(s) \\
s L_{2} i_{2}(s)=-D v_{1}(s)+D_{c} v(s)-\frac{V_{g}}{D_{c}} d(s) \\
\left(s C_{2}+\frac{1}{R}\right) v(s)=-D_{c} i_{2}(s)+D_{c} i_{g}(s)-\frac{I_{g}}{D} d(s)
\end{gathered}
$$

where $D$ is the duty cycle of the main switch $S$ in steady state operation, $D_{c}$ is the complement of the duty cycle $\left(D_{c}=1-D\right), V_{g}$ and $I_{g}$ are the dc values of the input voltage and current, $v_{g}(s)$ and $v(s)$ are the ac values of the input and output voltages, $i_{g}(s)$ is the input ac current, $v_{l}(s)$ represents the ac voltage over capacitor $C_{1}, i_{2}(s)$ is the ac current across inductor $L_{2}$ and $i(s)$ is the ac load current.

With these set of equations it is possible to arrive to the converter control-to-output $G_{v d}(s)$ and the converter line-tooutput $G_{v g}(s)$ voltage transfer functions, given by

$$
\begin{gathered}
G_{v g}(s)=R D_{c} \frac{L_{2} C_{1} s^{2}+D}{a_{4} s^{4}+a_{3} s^{3}+a_{2} s^{2}+a_{1} s+a_{0}} \\
G_{v d}(s)=\frac{R}{D} \frac{\left[-L_{1} L_{2} C_{1} I_{g} s^{3}+\left(L_{1}+L_{2}\right) C_{1} V_{g} D s^{2}-L_{1} I_{g} D s+V_{g} D\right]}{a_{4} s^{4}+a_{3} s^{3}+a_{2} s^{2}+a_{1} s+a_{0}}
\end{gathered}
$$

One can also obtain the duty ratio $d(s)$ to input current $i_{g}(s)$ transfer function $G_{i d}(s)$ and the input admittance $G_{i g}(s)$ as a quotient of the input current $i_{g}(s)$ and the input voltage $v_{g}(s)$, represented by

$$
\begin{gathered}
G_{i g}(s)=\frac{L_{2} C_{1} C_{2} R^{2} s^{3}+L_{2} C_{1} s^{2}+\left(C_{1} D_{c}^{2}+C_{2} D^{2}\right) R s+D^{2}}{a_{4} s^{4}+a_{3} s^{3}+a_{2} s^{2}+a_{1} s+a_{0}} \\
G_{i d}(s)=V_{g} \frac{L_{2} C_{1} C_{2} R^{2} s^{3}+L_{2}\left[\left(C_{1}+C_{2}\right) D+C_{1}\right] R s^{2}+\left(C_{2} R^{2}+L_{2}\right) D s+2 R D}{a_{4} s^{4}+a_{3} s^{3}+a_{2} s^{2}+a_{1} s+a_{0}}
\end{gathered}
$$

where the coefficients $a_{0}$ to $a_{4}$ in (9) to (12) are defined as

$$
\begin{gathered}
a_{0}=R D_{c}^{2} \\
a_{1}=L_{1} D^{2}+L_{2} D_{c}^{2} \\
a_{2}=R\left[\left(L_{1}+L_{2}\right) C_{1} D_{c}^{2}+\left(L_{1} D^{2}+L_{2} D_{c}^{2}\right) C_{2}\right] \\
a_{3}=L_{1} L_{2} C_{1} \\
a_{4}=L_{1} L_{2} C_{1} C_{2} R
\end{gathered}
$$

\section{DYNAMIC EQUIVALENT ELECTRICAL CIRCUIT OF PWM CCM DC-DC SEPIC CONVERTER}

Based on the dynamic equations (5) to (8) of the PWM CCM dc-dc Sepic converter, an initial electrical dynamic equivalent circuit can be obtained, as shown in Figure 3 [7].

Fig. 3. Initial equivalent dynamic circuit for PWM CCM dc-dc Sepic converter. 


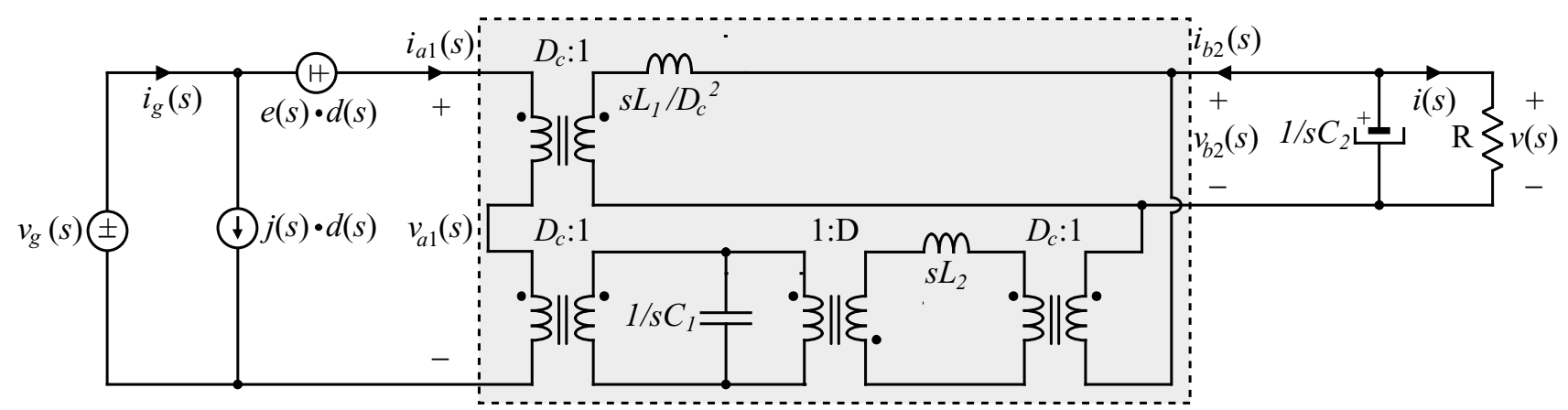

Fig. 4. Equivalent dynamic circuit for PWM CCM dc-dc Sepic converter as a two-port network.

Starting from the circuit presented in Figure 3 and reflecting the voltage and current sources for the input side of the circuit and the impedances to the load side and cascading ideal transformers when possible, one can arrives to a simplified circuit that cannot be further simplified by cascading the ideal remaining transformers, which are connected in a "circular way".

\section{THE PROPOSED APPROACH TO DERIVE THE CANONICAL EQUIVALENT CIRCUIT DYNAMIC MODEL FOR PWM CCM DC-DC SEPIC CONVERTER}

As already stressed, the $e(s) d(s)$ and $j(s) d(s)$ ac sources in the front-end of the canonical equivalent circuit (Figure 1) already account for the ac variations in the voltages and currents of the converter caused by control input variations $d(s)$. These ac variations are represented by the various ac voltage and current sources scattered in the initial equivalent dynamic circuit for PWM CCM dc-dc Sepic converter (Figure 3).

Thus, one can just ignore these sources and push inductor $L_{I}$ to the secondary side of the on-the-top ideal transformer. The equivalent dynamic circuit for the converter is re-draw in the sequence (Figure 4), where the frame for quadripole approach (dashed lines) and also the two-port voltages and currents $v_{a l}(s), v_{b 2}(s), i_{a l}(s)$ and $i_{b 2}(s)$ are already presented.

\section{TWO-PORT NETWORK ANALYSIS FOR THE PWM CCM DC-DC SEPIC CONVERTER}

It is possible to represent the framed part of the Sepic equivalent circuit (Figure 4) as an impedance two-port network with the relationships between voltages and currents in the input and output ports given by

$$
\left[\begin{array}{l}
v_{a 1}(s) \\
v_{b 2}(s)
\end{array}\right]=Z(s)\left[\begin{array}{l}
i_{a 1}(s) \\
i_{b 2}(s)
\end{array}\right]=\left(\begin{array}{ll}
z_{11}(s) & z_{12}(s) \\
z_{21}(s) & z_{22}(s)
\end{array}\right)\left[\begin{array}{l}
i_{a 1}(s) \\
i_{b 2}(s)
\end{array}\right]
$$

where $Z(s)$ is the impedance matrix with parameters $z_{11}(s)$, $z_{12}(s), z_{21}(s)$ and $z_{22}(s)$ [21].

Parameters $z_{11}(s)$ and $z_{21}(s)$ are obtained from (18) putting $i_{b 2}(s)=0$ and are given by

$$
\begin{aligned}
& z_{11}(s)=v_{a 1}(s) / i_{a 1}(s) \\
& z_{21}(s)=v_{b 2}(s) / i_{a 1}(s)
\end{aligned}
$$

Parameters $z_{22}(s)$ and $z_{12}(s)$ are obtained from (18) putting $i_{a l}(s)=0$ and are given by

$$
z_{22}(s)=v_{b 2}(s) / i_{b 2}(s)
$$

$$
z_{12}(s)=v_{a 1}(s) / i_{b 2}(s)
$$

Now, one must put $i_{a 1}(s)=0$ on the circuit presented in Figure 4 and parameters $z_{11}(s)$ and $z_{12}(s)$ are obtained by circuit analysis [21] and are defined by

$$
\begin{gathered}
z_{11}(s)=s\left(L_{1}+L_{2}\right)+\frac{1}{s C_{1}} \\
z_{21}(s)=\frac{s L_{2}}{D_{c}}+\frac{1}{s C_{1} \frac{D_{c}}{D}}
\end{gathered}
$$

Also, one must put $i_{b 2}(s)=0$ on the circuit presented in Figure 4 and parameters $z_{22}(s)$ and $z_{12}(s)$ are obtained by circuit analysis [21] and are defined by

$$
\begin{gathered}
z_{22}(s)=\frac{s L_{2}}{D_{c}^{2}}+\frac{1}{s C_{1}\left(\frac{D_{c}}{D}\right)^{2}} \\
z_{12}(s)=\frac{s L_{2}}{D_{c}}+\frac{1}{s C_{1} \frac{D_{c}}{D}}
\end{gathered}
$$

The resulting quadripole is reciprocal and, thus, $z_{21}(s)=$ $z_{12}(S)$.

One can convert the impedance matrix $Z(s)$ to the equivalent inverse transmission matrix $\operatorname{Tinv}(s)$ with the relationships between voltages and currents in the input and output ports given by

$$
\left[\begin{array}{l}
v_{b 2}(s) \\
-i_{b 2}(s)
\end{array}\right]=\operatorname{Tinv}(s)\left[\begin{array}{l}
v_{a 1}(s) \\
i_{a 1}(s)
\end{array}\right]=\left(\begin{array}{ll}
a_{11}(s) & a_{12}(s) \\
a_{21}(s) & a_{22}(s)
\end{array}\right)\left[\begin{array}{c}
v_{a 1}(s) \\
i_{a 1}(s)
\end{array}\right]
$$
with

with parameters $a_{11}(s), a_{12}(s), a_{21}(s)$ and $a_{22}(s)$ defined

$$
\begin{aligned}
& a_{11}(s)=\frac{z_{22}(s)}{z_{12}(s)} \\
& a_{12}(s)=-\frac{\Delta Z(s)}{z_{12}(s)} \\
& a_{21}(s)=-\frac{1}{z_{12}(s)} \\
& a_{22}(s)=\frac{z_{11}(s)}{z_{12}(s)}
\end{aligned}
$$

where $\Delta Z(s)$ means the determinant of matrix $Z(s)$.

Notice that this quadripole is also reciprocal. Therefore, $\Delta \operatorname{Tinv}(s)=a_{11}(s) a_{22}(s)-a_{12}(s) a_{21}(s)=1$, where $\Delta \operatorname{Tinv}(s)$ means the determinant of matrix $\operatorname{Tinv}(s)$. 


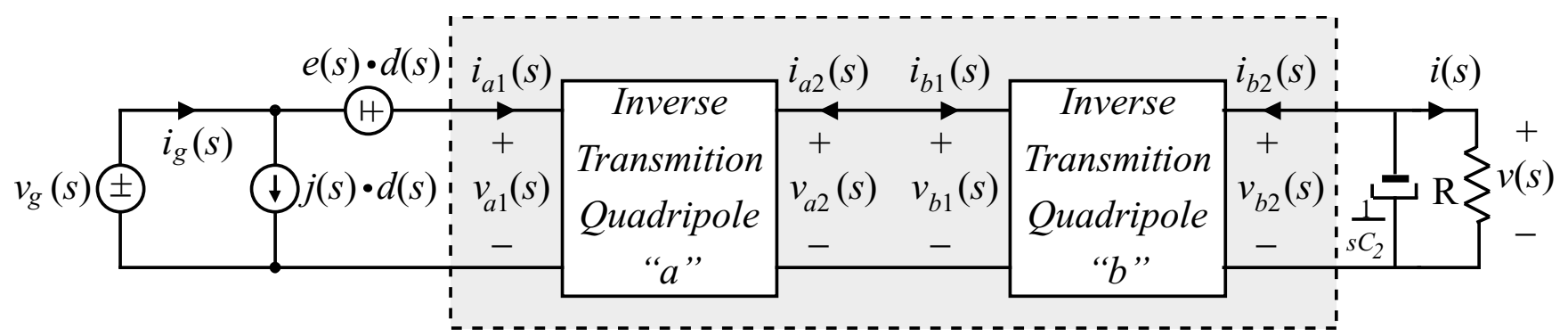

Fig. 5. Equivalent dynamical circuit for PWM CCM dc-dc Sepic converter as a two cascaded inverse transmission quadripoles.

Now, one can imagine that in this case the inverse transmission quadripole $\operatorname{Tinv}(s)$ shown in grey color in Figure 5, represents two cascaded quadripoles defined by matrices $\operatorname{Tinv}_{a}(s)$ and $\operatorname{Tinv}_{b}(s)$.

$\operatorname{Tinv}_{a}(s)$ represents the transformer of the canonical model, with conversion ratio $M(D)$. The conversion ratio $M(D)$ is equal to $D / D_{c}$ for the PWM CCM dc-dc Sepic converter. Thus, the relationships between matrices $\operatorname{Tinv}(s), \operatorname{Tinv}_{b}(s)$ and $\operatorname{Tinv}_{a}(s)$ can be defined by

$$
\begin{aligned}
\operatorname{Tinv}(s) & =\operatorname{Tinv}_{b}(s) \operatorname{Tinv}_{a}(s) \\
\operatorname{Tinv}_{b}(s) & =\operatorname{Tinv}(s) \operatorname{Tinv}(s)^{-1}
\end{aligned}
$$

where:

$$
\operatorname{Tinv}_{a}(s)=\left(\begin{array}{cc}
D / D_{c} & 0 \\
0 & D_{c} / D
\end{array}\right)
$$

Operating (33) with $\operatorname{Tinv}(s)$ and $\operatorname{Tinv}_{a}(s)$ given by (32) and (34), respectively, one arrives to

$$
\operatorname{Tinv}_{b}(s)=\left(\begin{array}{ll}
a_{b 11}(s) & a_{b 12}(s) \\
a_{b 21}(s) & a_{b 22}(s)
\end{array}\right)=\left(\begin{array}{ll}
\frac{D_{c}}{D} a_{11}(s) & \frac{D}{D_{c}} a_{12}(s) \\
\frac{D_{c}}{D} a_{21}(s) & \frac{D}{D_{c}} a_{22}(s)
\end{array}\right)
$$

The next step is re-convert $\operatorname{Tinv}_{b}(s)$ into an impedance quadripole matrix $Z_{b}(s)$

$$
Z_{b}(s)=\left(\begin{array}{cc}
z_{b 11}(s) & z_{b 12(s)} \\
z_{b 21}(s) & z_{b 22(s)}
\end{array}\right)=\left(\begin{array}{rr}
-\frac{a_{b 22}(s)}{a_{b 21}(s)} & -\frac{1}{a_{b 21}(s)} \\
-\frac{\Delta \operatorname{Tinv}_{b}}{a_{b 21}(s)} & -\frac{a_{b 11}(s)}{a_{b 21}(s)}
\end{array}\right)
$$

where $\Delta \operatorname{Tinv}_{b}(s)$ means the determinant of $\operatorname{Tinv}_{b}(s)$ matrix.

One can observe that the quadripole $\operatorname{Tinv}_{b}(s)$ is also reciprocal and so the matrix determinant $\Delta \operatorname{Tinv}_{b}(s)$ in (36) equals 1 .

Now, remembering that the $Z_{b}(s)$ quadripole is also reciprocal, thus $z_{b 21}(s)=z_{b 12}(s)$ it is possible to represent the new impedance parameters by

$$
\begin{gathered}
z_{b 11}(s)=s\left(L_{1}+L_{2}\right)\left(\frac{D}{D_{c}}\right)^{2}+\frac{1}{s C_{1}\left(\frac{D_{c}}{D}\right)^{2}} \\
z_{b 22}(s)=\frac{s L_{2}}{D_{c}^{2}}+\frac{1}{s C_{1}\left(\frac{D_{c}}{D}\right)^{2}}
\end{gathered}
$$

$$
z_{b 12}(s)=z_{b 21}(s)=\frac{s L_{2} D}{D_{c}^{2}}+\frac{1}{s C_{1}\left(\frac{D_{c}}{D}\right)^{2}}
$$

Finally, the $Z_{b}(s)$ quadripole can be represented by an equivalent circuit shown in Figure 6, where $z_{A}(s), z_{B}(s)$ and $z_{C}(s)$ are

$$
\begin{gathered}
z_{A}(s)=z_{b 11}(s)-z_{b 12}(s)=s\left(\frac{L_{1} D^{2}-L_{2} D D_{c}}{D_{c}^{2}}\right)=s L_{A} \\
z_{B}(s)=z_{b 22}(s)-z_{b 12}(s)=s \frac{L_{2}}{D_{c}}=s L_{B} \\
z_{C}(s)=z_{b 12}(s)=\frac{s L_{2} D}{D_{c}^{2}}+\frac{1}{s C_{1}\left(\frac{D_{c}}{D}\right)^{2}}=S L_{C}+\frac{1}{s C_{C}}
\end{gathered}
$$

where $s L_{A}, s L_{B}, s L_{C}$ and $1 / s C_{C}$ represent equivalent inductive and capacitive reactances.

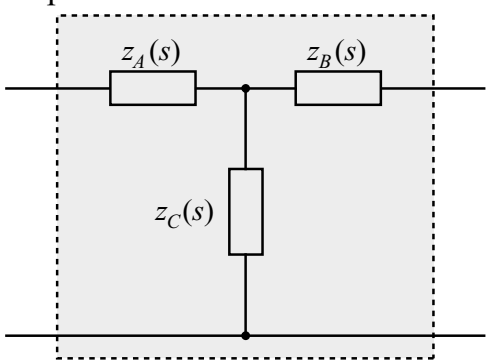

Fig. 6. Equivalent circuit for the $\mathrm{Zb}(\mathrm{s})$ quadripole.

As a last step, one must just insert the transformer of the canonical model cascaded with the equivalent circuit for the $Z_{b}(s)$ quadripole in the framed block (dashed lines) of the equivalent dynamic circuit for PWM CCM dc-dc Sepic converter (Figure 4). With this, it is recognized that the lowpass filter includes capacitor $C_{2}$ as presented in Figure 7, which shows the complete canonical ac model of this converter.

One can observe that this low-pass filter contains five energy storage elements (inductors $L_{A}, L_{B}, L_{C}$ and capacitors $C_{C}$ and $C_{2}$ ) but, even though, it is a fourth-order one.

In order to verify the equivalence, one just need to analyze the resulting electric circuit shown in Figure 7 putting $v_{g}(s)=0$ to obtain the same expression for $G_{v d}(s)$ as presented in equation (10). Also, putting to zero the sources $e(s) . d(s)$ and $j(s) . d(s)$, on will arrive to the same expression for $G_{v g}(s)$ as presented in equation (9). For these reason, it seems unnecessary to plot a Bode diagram to compare those transfer functions. 


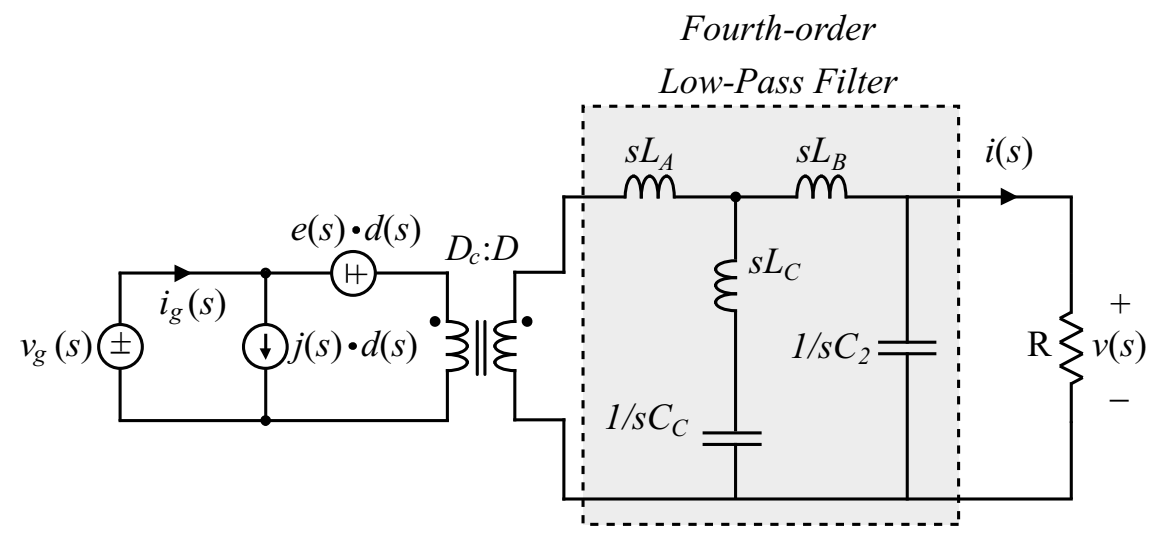

Fig. 7. The complete canonical small signal \& low frequency ac model of the PWM CCM dc-dc Sepic converter.

Fourth-order

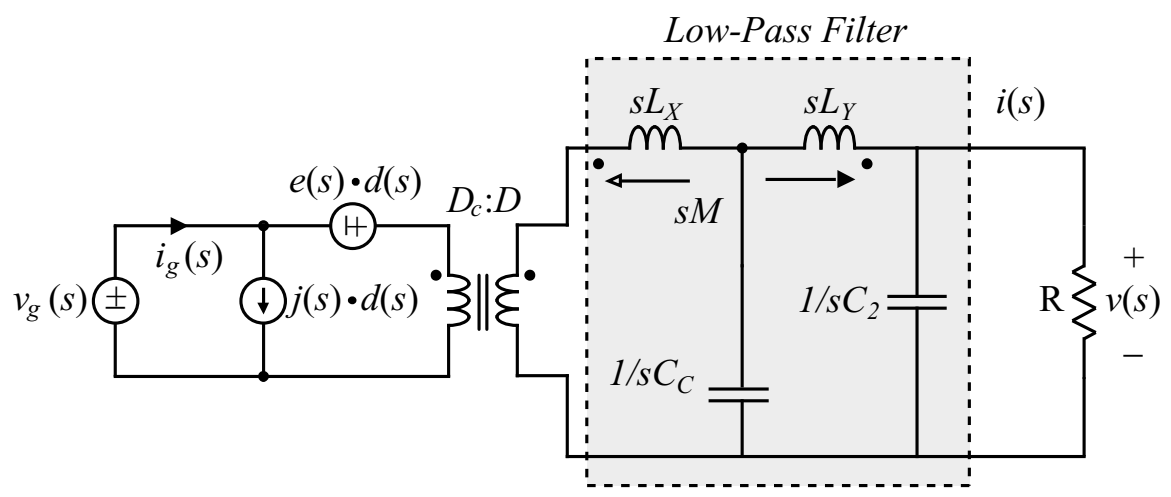

Fig. 8. The complete canonical small signal \& low frequency ac model of the PWM CCM dc-dc Sepic converter with coupled inductors.

It is also possible represent inductors $L_{A}, L_{B}, L_{C}$ in Figure 7 as two coupled inductors $L_{X} \& L_{Y}$, with mutual inductance $M$ [20] and re-draw the canonical circuit as shown in Figure 8.

In this case

$$
\begin{gathered}
M=L_{C}=\frac{L_{2} D}{D_{c}^{2}} \\
L_{X}=L_{A}+M=\frac{\left(L_{1}+L_{2}\right) D^{2}}{D_{c}^{2}} \\
L_{Y}=L_{B}+M=\frac{L_{2}}{D_{c}^{2}} \\
k=\frac{M}{\sqrt{L_{X} L_{Y}}}=\sqrt{\frac{L_{2}}{L_{1}+L_{2}}}
\end{gathered}
$$

where $k$ is the magnetic coupling coefficient $(k<1)$.

One can notice that for the Sepic converter, impedance $z_{A}(s)$ (expression (40) and Figure 7) may become negative, depending on the duty cycle $D$. In this case, the effect of an internal negative inductance belonging to the fourth-order low-pass filter is emulated.

Impedance $z_{A}(s)$ become null when $L_{2}=L_{1} D / D_{c}$ and the output low-pass filter become a second-order one, with the converter line-to-output $G_{v g}(s)$ voltage transfer functions, now given by

$$
G_{v g}(s)=R D D_{c} \frac{1}{L_{1} C_{2} R D s^{2}+L_{1} D s+R D_{c}}
$$

In this case the magnetic coupling coefficient $k(k<1)$ is given by

$$
k=\frac{M}{\sqrt{L_{X} L_{Y}}}=\sqrt{D}
$$

This fact may be interesting and be subject to further investigation.

\section{THE COMPLETE CANONICAL SMALL SIGNAL \& LOW FREQUENCY AC MODEL OF THE ZETA CONVERTER}

Repeating the same procedure for the PWM CCM dc-dc Zeta converter shown in Figure 9, the complete canonical small signal \& low frequency ac model of this converter can also be obtained, and is topologically equal to the ac model of the Sepic converter shown in Figure 7.

As it can be seen, the basic topology of the low pass fourth-order filter is the same for those two converters, but for Zeta converter, $z_{A}(s), z_{B}(s)$ and $z_{C}(s)$ are given by

$$
\begin{gathered}
z_{A}(s)=s\left(\frac{L_{1} D}{D_{c}^{2}}\right)=s L_{A} \\
z_{B}(s)=s \frac{\left(L_{2} D_{c}-L_{1} D\right)}{D_{c}}=s L_{B} \\
z_{C}(s)=\frac{s L_{1}}{D_{c}}+\frac{1}{s C_{1}}=S L_{C}+\frac{1}{s C_{C}}
\end{gathered}
$$

where $s L_{A}, s L_{B}, s L_{C}$ and $1 / s C_{C}$ represent equivalent inductive and capacitive reactances. 


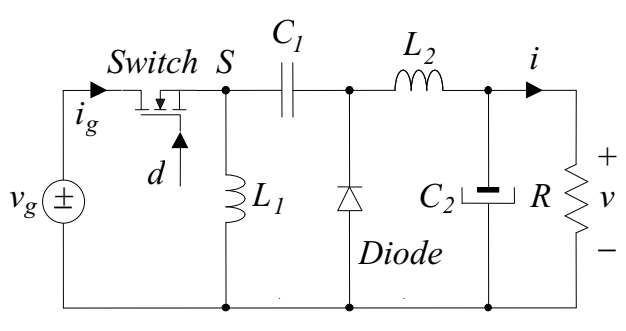

Fig. 9. Zeta PWM CCM dc-de converter.

It is also possible represent inductors $L_{A}, L_{B}, L_{C}$ as two coupled inductors $L_{X} \& L_{Y}$, with mutual inductance $M$ [20] as already made for the Sepic converter (Section VI).

One can notice that for the Zeta converter, impedance $z_{B}(s)$ may become negative, depending on the duty cycle $D$, showing that in this case either, the effect of an internal negative inductance belonging to the fourth-order low-pass filter is emulated.

Impedance $z_{B}(s)$ become null when $L_{2}=L_{1} D / D_{c}$ and the output low-pass filter become a second-order one. As for the Sepic converter, further investigation concerning this fact may be interesting.

\section{CONCLUSION}

The complete canonical small signal \& low frequency ac model of PWM CCM dc-dc Sepic and Zeta converters are obtained in a simple manner, by employing the two-port network theory. Even if this work deals with ideal elements, the inclusion of non-idealities of inductors and capacitors (resistances, for instance) as well as the switches losses, do not represent a burden for analysis.

It was demonstrated that with a unique approach, one can obtain, in an easy way, the complete canonical small signal \& low frequency ac model of classic PWM CCM dc-dc converters and this approach may also be applied to converter analysis and modeling of improved topology converters (for instance, [19]) or even to converters based on generic switching cells such as in [9].

The obtained equivalent circuit indicates the possibility to operate the Sepic and the Zeta converter emulating a second order low-pass output filter, depending on parameters $L_{1}, L_{2}$ and $D$. The consequences of that possibility may be object of further investigation.

\section{REFERENCES}

[1] R. D. Middlebrook and S. Cuk, A general unified approach to modeling switching-converter power stages, IEEE Power Electronics Specialists Conference, 1975 Record, pp. 80-90.

[2] S. Cuk, Modeling, analysis, and design of switching converters, $\mathrm{Ph}$. D. thesis, California Institute of Technology, USA, November 1976.

[3] V. Vorperian, Simplified analysis of PWM converters using the model of the PWM switch: Parts I and II, IEEE Transactions on Aerospace and Electronic Systems, Vol. AES-26, pp. 490-505, May 1990.

[4] S. R. Sanders, J. M. Noworolski, X. Z. Liu and G. C. Verghese, Generalized averaging method for power conversion circuits, IEEE Power Electronics Specialists Conf, San Antonio - USA, June 1990.

[5] A. S. Kislovski, R. Redl and N. O. Sokal, Dynamic analysis of switching-mode $d c / d c$ converters, Van Nostrand Reinhold, New York - USA, 1991.

[6] E. Van Dijk, H. J. N. Spruijt, D. M. O'Sullivan and J. B. Klaassens, PWM-switch modeling of $d c-d c$ converters, IEEE Transactions on Power Electronics, Vol. 10, No. 6, November 1995.

[7] R. W. Erickson, Fundamentals of power electronics, Chapman \& Hall, 1997.

[8] R. D. Middlebrook, Small-signal modeling of pulsewidth modulated switched-mode power converters, proceedings of the IEEE, Vol. 76, No. 4, April 1998.

[9] T. Wu and Y. Chen, Modeling PWM dc/dc converters out of basic converters units, IEEE Transactions on Power Electronics, Vol. 13, No. 5, September 1998.

[10] A. J. Forsyth and S. V. Mollov, Modelling and control of $d c-d c$ converters, Power Engineering Journal, October 1998.

[11]E. Niculescu and E. P. Iancu, Modeling and analysis of the $d c / d c$ fourth-order converters, $7^{\text {th }}$ Workshop on Computers in Power Electronics, pp. 83-88, Blacksburg, VA, USA, July 2000

[12]D. Maksimovic, Computer-aided small-signal analysis based on impulse response of $d c / d c$ switching power converters, IEEE Transactions on Power Electronics, Vol. 15, No. 6, November 2000.

[13]D. Maksimovic, A. M. Stankovic, V. J. Thottuvelil and G. C. Verghese, Modeling and simulation of power electronic converter, Proceedings of IEEE, Vol. 89, No. 6, June 2001.

[14] J. Chen, R. W. Erickson and D. Maksimovic, Averaged switch modeling of boundary conduction mode dc-to-dc converters, IECON'01: The 27th Annual Conference of the IEEE Industrial Electronics Society.

[15]P. G. Maranesi and M. Riva, Automatic modeling of $P W M d c-d c$ converters, IEEE Power Electronics Letters, Vol. I, No. 4, December 2003.

[16]W. Gu, Small signal modeling for current mode controlled Cuk and Sepic converters, APEC 2005 Applied Power Electronics Conference and Exposition, 2005, pp. 906-910, vol. 2.

[17] A. Hren and P. Slibar, Full order dynamic model of Sepic converter, IEEE ISIE 2005, June 20-23, 2005, Dubrovnik, Croatia.

[18]A. Davoudi, J. Jatskevich and T. De Rybel, Numerical state-space average-value modeling of PWM $d c-d c$ converters operating in DCM and CCM, IEEE Transactions on Power Electronics, Vol. 21, No. 4, July 2006.

[19] M. A. Al-Saffar, E. H. Ismail, A. J. Sabzali and A. A. Fardoun, An improved topology of Sepic converter with reduced output voltage ripple, IEEE Transactions on Power Electronics, Vol. 23, No. 5, September 2008.

[20] M. L. Heldwein, EMC filtering of three-phase PWM converters, Sudwestdeutscher Verlag fur Hochschulschriften Aktiengesellschaft \& Co. KG, Germany, 2009.

[21] J. W. Nilsson and S. A. Riedel, Electric Circuits, Addison-Wesley Publishing Company, 1996. 


\section{BIOGRAPHY}

Enio V. Kassick was born in Taquara RS in ' 56 and earned his engineering degree in ' 80 at Federal University of Rio Grande do Sul (UFRGS-Brazil), his M. Sc degree in '83 at Federal University of Santa Catarina (UFSC-Brazil) and his Dr. degree in 90 at Institut National Polytechnique de Toulouse (INPT-France). His is with INEP - Power Electronics Institute of the Federal University of Santa Catarina since ` 81 . In ` 83 he started his academic career at UFSC as a lecturer in the Electrical Engineering Dept and became a professor in ' 99 . His research interests include power electronics and power quality.

Dr. Kassick is currently a member of the Brazilian Power Electronics Society (SOBRAEP). 